The University of Southern Mississippi

The Aquila Digital Community

Faculty Publications

$6-4-2008$

\title{
A Boundary Meshless Method Using Chebyshev Interpolation and Trigonometric Basis Function for Solving Heat Conduction Problems
}

\author{
S. Yu. Reutskiy \\ The National Academy of Science of Ukraine \\ C.S. Chen \\ University of Southern Mississippi, CS.Chen@usm.edu \\ Haiyan Y. Tian \\ University of Southern Mississippi, Haiyan.Tian@usm.edu
}

Follow this and additional works at: https://aquila.usm.edu/fac_pubs

Part of the Physical Sciences and Mathematics Commons

\section{Recommended Citation}

Reutskiy, S. Y., Chen, C., Tian, H. Y. (2008). A Boundary Meshless Method Using Chebyshev Interpolation and Trigonometric Basis Function for Solving Heat Conduction Problems. International Journal for Numerical Methods in Engineering, 74(10), 1621-1644.

Available at: https://aquila.usm.edu/fac_pubs/1513

This Article is brought to you for free and open access by The Aquila Digital Community. It has been accepted for inclusion in Faculty Publications by an authorized administrator of The Aquila Digital Community. For more information, please contact Joshua.Cromwell@usm.edu. 


\begin{tabular}{|c|c|c|}
\hline NME 2230 & PROD TYPF. COM & ED: Vijaya \\
\hline pp: 1-24 (col.fig.: Nil) & & PAGN: Veena - SCAN: Monica \\
\hline
\end{tabular}

INTERNATIONAL JOURNAL FOR NUMERICAL METHODS IN ENGINEERING

Int. J. Numer. Meth. Engng (2007)

Published online in Wiley InterScience (www.interscience.wiley.com). DOI: 10.1002/nme.2230

\title{
A boundary meshless method using Chebyshev interpolation and 3 trigonometric basis function for solving heat conduction problems
}

\author{
S. Yu. Reutskiy ${ }^{1}$, C. S. Chen ${ }^{2, * \dagger}{ }^{\dagger}$ and H. Y. Tian ${ }^{2}$ \\ $5{ }^{1}$ Science and Technology Center of Magnetism of Technical Objects, The National Academy of Science of Ukraine, \\ Industrialnaya St.,19, 61106, Kharkov, Ukraine \\ ${ }^{2}$ Department of Mathematics, University of Southern Mississippi, Hattiesburg, MS 39406, U.S.A.
}

\section{SUMMARY}

9 A boundary meshless method has been developed to solve the heat conduction equations through the use of a newly established two-stage approximation scheme and a trigonometric series expansion scheme to 11 approximate the particular solution and fundamental solution, respectively. As a result, no fundamental solution is required and the closed form of approximate particular solution is easy to obtain. The effective-

13 ness of the proposed computational scheme is demonstrated by several examples in 2D and 3D. We also compare our proposed method with the finite-difference method and the other meshless method showed

15 in Šarler and Vertnik (Comput. Math. Appl. 2006; 51:1269-1282). Excellent numerical results have been observed. Copyright (C) 2007 John Wiley \& Sons, Ltd.

Received 8 February 2007; Revised 23 July 2007; Accepted 28 September 2007

17 KEY WORDS: method of fundamental solutions; particular solution; Chebyshev interpolation; $C$-expansion; diffusion equations

\section{INTRODUCTION}

During the past decade, meshless methods have attracted great attention in the area of scientific

21 computing. Various types of numerical techniques for solving science and engineering problems without domain discretization have been developed. One of the common goals of developing meshless methods is to solve a given set of partial differential equations (PDEs) with minimum human and computational costs. Hence, other than the accuracy and efficiency, the simplicity of the implementation of the developed meshless algorithm is also of great importance.

Among all the proposed meshless methods, Trefftz-type methods have been vigorously reinvestigated in recent years. A special type of Trefftz method is the method of fundamental solutions

\footnotetext{
*Correspondence to: C. S. Chen, Department of Mathematics, University of Southern Mississippi, Hattiesburg, MS 39406, U.S.A.

†E-mail: cschen.math@gmail.com
} 
1 (MFSs) which has been extended to solve various elliptic and time-dependent problems [1-3]. However, the fundamental solution of a given differential equation is not always available. The

3 ill conditioning of the MFS and the location of source points are also issues to be resolved. As a result, despite the effectiveness of the MFS, its applicability is somehow limited. To alleviate these

5 difficulties, we introduce the method of approximate fundamental solutions (MAFSs) [4] in which the trial function is approximated by the truncated trigonometric series. In this way, the fundamental

7 solution of the given partial differential operator is not required. For non-homogeneous equation, getting a closed form particular solution is not a trivial task $[2,3]$. Recently, a novel and effective

9 numerical technique for approximating the particular solutions of a new class of differential equations has been developed [5]. It combines the advantages of polynomial interpolation and

11 trigonometric approximation to the source function so that the closed form approximate particular solution can be easily and accurately obtained. This technique includes two major steps: (i)

13 approximating the source function using Chebyshev polynomials, (ii) Chebyshev interpolants are further approximated by a C-Expansion approximation scheme, a trigonometric-based scheme.

15 One of the advantages of this technique is that the closed form of approximate particular solution can be easily obtained. This approach is also highly accurate due to the spectral convergence of

17 Chebyshev interpolation. We would like to note that such a two-stage approximation scheme is not necessary from the point of view of pure function approximation. However, our ultimate goal is

19 to develop an approximation scheme so that the approximate particular solution can be evaluated efficiently for a more general class of differential operators. Furthermore, it is interesting that an 21 approximate fundamental solution can be obtained in a way similar to the derivation of particular solutions using the same trigonometric basis functions [4,5]. Encouraged by the success of these 23 novel approaches for solving elliptic problems [5], we extend these techniques to solve transient heat conduction problems.

25 There are various numerical approaches to solving heat conduction problems. The common approaches are (i) time-space separation [6]; (ii) Laplace transform or Fourier transform to remove 27 the time dependence $[7,8]$; (iii) time difference scheme $[9,10]$. There are advantages and disadvantages in each approach. Apparently, the time difference scheme is the most popular approach 29 being applied for solving time-dependent problems. In this paper, we will focus on this approach.

We consider the following initial boundary value problem

$$
\begin{aligned}
\frac{\partial u(\mathbf{x}, t)}{\partial t} & =L[u(\mathbf{x}, t)]+f(\mathbf{x}, t), \quad \mathbf{x} \in \Omega \subset \mathbb{R}^{d}, \quad t>0, \quad d=1,2,3 \\
B[u(\mathbf{x}, t)] & =g(\mathbf{x}, t), \quad \mathbf{x} \in \partial \Omega \\
u(\mathbf{x}, 0) & =u_{0}(\mathbf{x}), \quad \mathbf{x} \in \Omega
\end{aligned}
$$

31 where $L$ is a time-independent linear differential operator, $B$ is a boundary operator, and $\Omega$ is a simply connected domain bounded by a simple closed curve $\partial \Omega$.

33 Finite difference in time transforms (1)-(3) to a sequence of elliptic equations. Using the likewise Crank-Nicholson $(\mathrm{C}-\mathrm{N})$ scheme with the second order approximation in time

$$
\frac{u^{j+1}(\mathbf{x})-u^{j}(\mathbf{x})}{\Delta t}=\frac{1}{2}\left[L\left[u^{j+1}(\mathbf{x})\right]+L\left[u^{j}(\mathbf{x})\right]\right]+f^{j+1 / 2}(\mathbf{x})
$$


we obtain a sequence of inhomogeneous equations

$$
\begin{aligned}
(L-p)\left[u^{j+1}(\mathbf{x})\right] & =-(L+p)\left[u^{j}(\mathbf{x})\right]-2 f^{j+1 / 2}(\mathbf{x}), \quad \mathbf{x} \in \Omega, \quad j=0,1,2, \ldots \\
B\left[u^{j+1}(\mathbf{x})\right] & =g^{j+1}(\mathbf{x}), \quad \mathbf{x} \in \partial \Omega
\end{aligned}
$$

where $u^{j}(\mathbf{x})=u\left(\mathbf{x}, t^{j}\right), f^{j+1 / 2}(\mathbf{x})=f\left(\mathbf{x},\left(t^{j}+t^{j+1}\right) / 2\right), g^{j+1}(\mathbf{x})=g\left(\mathbf{x}, t^{j+1}\right), t^{j}=j \Delta t, \Delta t$ is a 3 time step, and $p=2 / \Delta t$. The boundary value problem (4)-(5) can be solved by the method of particular solution in which an effective way of evaluating an approximate particular solution is crucial. We refer the readers to the references in $[2,3]$ for further details for the evaluation of particular solutions. In this paper, we employ the newly established Chebyshev interpolation and $C$-Expansion approximation scheme, which has been recently published in this journal, to evaluate the approximate particular solution [5].

9 For a homogeneous equation, many boundary meshless methods can be applied [2, 11]. According to the MAFS, an approximate solution of (4) at the $j+1$ th time step can be expressed 11 in the form

$$
u^{j+1}(\mathbf{x})=u_{p}^{j+1}(\mathbf{x})+\sum_{k=1}^{K} a_{k}^{j+1} \Psi_{k}(\mathbf{x})
$$

13 where $u_{p}^{j+1}(\mathbf{x})$ is a particular solution at the $j+1$ th time layer, and $\Psi_{k}(\mathbf{x}), k=1, \ldots, K$ are the approximate fundamental solutions. Note that, if differential operators of the form

$$
L=\sum_{k_{1}, k_{2}=0}^{l} A_{k_{1}, k_{2}} \frac{\partial^{2 k_{1}+2 k_{2}}}{\partial x_{1}^{2 k_{1}} \partial x_{2}^{2 k_{2}}}, \quad A_{k_{1}, k_{2}}=\text { const. }
$$

are considered and if the right-hand side of (4) is approximated by the trigonometric series, e.g.

$$
-(L+p)\left[u^{j}(\mathbf{x})\right]-2 f^{j+1 / 2}(\mathbf{x}) \simeq \sum_{n=1}^{M} \sum_{m=1}^{M} H_{n, m}^{(j)} \sin \left(n \pi \frac{x+1}{2}\right) \sin \left(m \pi \frac{y+1}{2}\right)
$$

then the particular solution $u_{p}^{j+1}$ can be written in the analytic form.

19 In the MAFS, the trial functions $\Psi_{k}(\mathbf{x})$ in (6) satisfy $(L-p)\left[\Psi_{k}\right]=I\left(x, y, \xi_{k}, \eta_{k}\right)$, where $I\left(x, y, \xi_{k}, \eta_{k}\right)$ is a $2 \mathrm{D}$ delta-shaped function, in the infinite domain. The detailed formulation of 21 the MAFS will be given in the next section.

The organization of this paper is as follows. In Section 2, we briefly introduce the basis functions 23 of the MAFS and provide three regularization methods for the formulation of MAFS. In Section 3, a finite-difference time-stepping scheme is employed to reduce the given heat conduction problem

25 to a sequence of modified Helmholtz equations. In Section 4, the method of particular solution has been employed to solve the modified Helmholtz equation for each time step. To demonstrate

27 the effectiveness of the proposed approach in this paper, numerical examples of heat conduction problems in regular and irregular domains in 2D and 3D are given in Section 5. 


\section{BASIS FUNCTIONS OF THE MAFS}

In this section, we briefly introduce the formulation of trial function using MAFs. The Laplace operator can be written as the sum of two 1D operators

$$
\nabla^{2}=-l^{(x)}-l^{(y)}, \quad l^{(x)}=-\frac{\partial^{2}}{\partial x^{2}}, \quad l^{(y)}=-\frac{\partial^{2}}{\partial y^{2}}
$$

5 We use minus sign before the operators in order to obtain a positive spectra.

We start the construction of the MAFS basis functions with the formal Fourier series for Dirac's

7 delta function. It is well known that the eigenfunctions

$$
\varphi_{n}(x)=\sin \left(\lambda_{n}(x+1)\right), \quad \lambda_{n}=\frac{n \pi}{2}, \quad n=1,2, \ldots
$$

9 are the solutions of the following Sturm-Liouville problem on the interval $[-1,1]$ :

$$
l^{(x)} \varphi=\lambda^{2} \varphi, \quad \varphi(-1)=\varphi(1)=0
$$

11 The eigenfunctions $\varphi_{n}(x)$ form an orthogonal system on $[-1,1]$ with the scalar product

$$
\int_{-1}^{1} \varphi_{n}(x) \varphi_{m}(x) \mathrm{d} x=\delta_{n, m}= \begin{cases}0, & m \neq n \\ 1, & m=n\end{cases}
$$

13 Thus, Dirac's delta function can be formally expressed as follows:

$$
\delta(x-\xi)=\sum_{n=1}^{\infty} \varphi_{n}(\xi) \varphi_{n}(x)
$$

15 Note that this series diverges at any point in the interval $[-1,1]$. With various kinds of regularization techniques, a smooth delta-shaped function, $I(x, \xi)$, can be constructed through the formal series

17 expansion (11); i.e. the regularized delta-shaped functions have the form

$$
I(x, \xi)=\sum_{n=1}^{M} r_{n}(M, \gamma) \varphi_{n}(\xi) \varphi_{n}(x)
$$

19 Note that $r_{n}(M, \gamma)$ is the regularization factor that can be obtained by the following regularization techniques:

21 1. The Lanczos regularization technique:

$$
r_{n}(M, \gamma)=\left[\sigma_{n}(M)\right]^{\gamma}, \quad \sigma_{n}(M)=\frac{\sin [v(n, M)]}{v(n, M)}, \quad v(n, M)=\frac{n \pi}{M+1}
$$

where $\sigma_{n}(M)$ are called the Lanczos sigma factors that are used to overcome Gibb's phenomenon in the Fourier series expansion of non-smooth functions [12]. This technique was employed in $[4,13]$ for solving stationary and time-dependent problems. The parameters $M$ and $\gamma$ should be taken in coupling. In all the calculations presented in this paper, we use $\gamma=4,6,8,12,14,16,18$ for $M=10,20,30,40,50,80,100$. This choice of the regularization parameter is found to be close to the optimal one. 
2. The Riesz regularization technique:

$$
r_{n}(M, \gamma)=\left(1-\frac{\lambda_{n}^{2}}{\lambda_{M+1}^{2}}\right)^{\gamma}
$$

This was proposed in [14] for solving elliptic PDEs with scattered data in irregular domains. 3. The Abel regularization technique: Let us consider the following equation:

$$
\frac{\partial w(t, x, \xi)}{\partial t}=\frac{\partial^{2} w(t, x, \xi)}{\partial x^{2}}
$$

with the initial distribution

$$
w(0, x, \xi)=\delta(x-\xi)=\sum_{n=1}^{\infty} \varphi_{n}(\xi) \varphi_{n}(x)
$$

We consider diffusion of the initial delta distribution. Let us look for a solution in the same form of the series

$$
w(t, x, \xi)=\sum_{n=1}^{\infty} w_{n}(t) \varphi_{n}(\xi) \varphi_{n}(x)
$$

From (15), we obtain

$$
w_{n}(t)=\exp \left(-\lambda_{n}^{2} t\right)
$$

for all $x, \xi$. We set the regularizing coefficients in the following way:

$$
r_{n}(\alpha)=\exp \left(-\alpha \lambda_{n}^{2}\right)
$$

i.e. $\alpha$ is the time moment in which we consider $w(t, x, \xi)$. This summation is also known as a heat-kernel regularized sum or a generalized Dirichlet series [15].

In the practical applications we use the truncated series

$$
I(x, \xi)=\sum_{n=1}^{M} r_{n}(\alpha) \varphi_{n}(\xi) \varphi_{n}(x)
$$

where $r_{M+1}(\alpha)=\varepsilon$ is a small prescribed value. In all the numerical results presented in this paper, we use $\alpha=0.005-0.01$ for $M=30, \alpha=0.001-0.005$ for $M=50$, and $\alpha=0.0012-0.0015$ for $M=100$.

The graph of 1D smooth approximations $I(x, \xi)$ of Dirac's delta function using the Lanczos 25 regularization techniques is shown in Figure 1. The graphs of $I(x, \xi)$ using the Riesz and Abel regularizations are similar to that in Figure 1. Note that we place here the graphics of the scaled

27 values $I(x, \xi) / I(\xi, \xi)$. As shown in the figure, $I(x, \xi)$ can approximate Dirac's delta function $\delta(x-\xi)$ as closely as we want by properly choosing the regularization factors. One important

29 distinction between these two functions is that $I(x, \xi) \in C^{\infty}$ while $\delta(x-\xi)$ is not a differentiable function. 


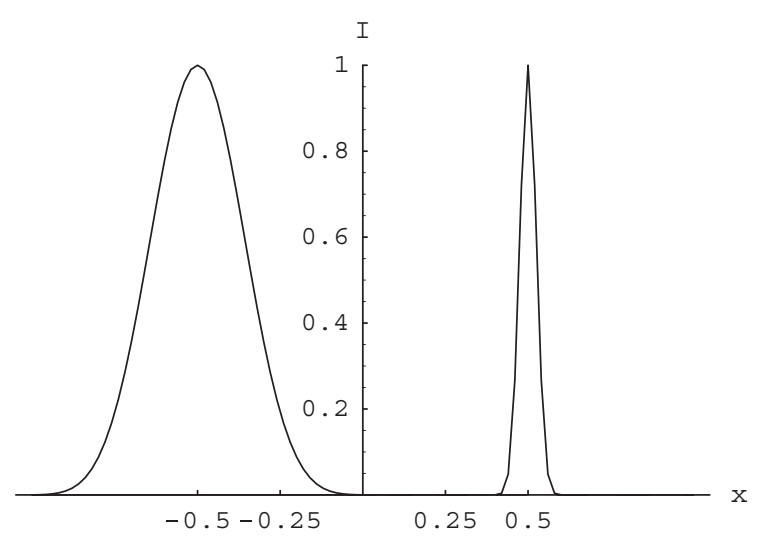

Figure 1. The Lanczos regularization. Approximations of the delta-function with $M=20$, $l=6$ (left) and $M=200, l=18$ (right).

The 2D delta-shaped functions can be obtained through the tensor product of the 1D ones; i.e.

$$
I(x, y, \xi, \eta)=I(x, \xi) I(y, \eta)=\sum_{n, m=1}^{M} c_{n, m}(\xi, \eta) \varphi_{n}(x) \varphi_{m}(y)
$$

3 where the coefficients $c_{n, m}(\xi, \eta)$ depend on the regularizing technique used.

In Figure 2, we plot the graphs of 2D delta-shaped basis functions $I(x, y, \xi, \eta)$ using Abel's

5 regularization technique with $M=20, \alpha=0.01$ and $M=200, \alpha=0.0003$, respectively. They are infinitely differentiable and are not 'identical' to zero in any interval. However, by visual obser-

7 vation, $I(x, y, \xi, \eta)$ differs from zero only inside some neighborhood of the center point $(\xi, \eta)$. In a way, $I(x, y, \xi, \eta)$ can be characterized as 'approximate locally supported functions'.

9 Regardless of the type of regularization technique, all the approximations $I(x, y, \xi, \eta)$ have the form of a truncated series over $\varphi_{n}(x) \varphi_{m}(y)$. The approximate fundamental solution $\Psi(x, y, \xi, \eta)$

11 of a given PDE can be obtained by using $I(x, y ; \xi, \eta)$ as the forcing term. For example, for the modified Helmholtz equation, we have

$$
\left(\nabla^{2}-p\right) \Psi(x, y, \xi, \eta)=I(x, y, \xi, \eta)
$$

Technically, $\Psi(x, y, \xi, \eta)$ in (21) is not only an approximate fundamental solution, but also a

15 particular solution. Since $I(x, y, \xi, \eta)$ is a linear combination of trigonometric functions, the particular solution $\Psi(x, y, \xi, \eta)$ in (21) has to be a linear combination of trigonometric functions also. Hence, $\Psi(x, y, \xi, \eta)$ has to be in the following form:

$$
\Psi(x, y, \xi, \eta)=\sum_{m, n=1}^{M} D_{n, m}(\xi, \eta) \varphi_{n}(x) \varphi_{m}(y)
$$

19 where $D_{n, m}$ are to be determined. Substituting (20) and (22) in (21), by the method of undetermined coefficients, we have

$$
D_{n, m}(\xi, \eta)=-\frac{c_{n, m}(\xi, \eta)}{\lambda_{n}^{2}+\lambda_{m}^{2}+p}
$$




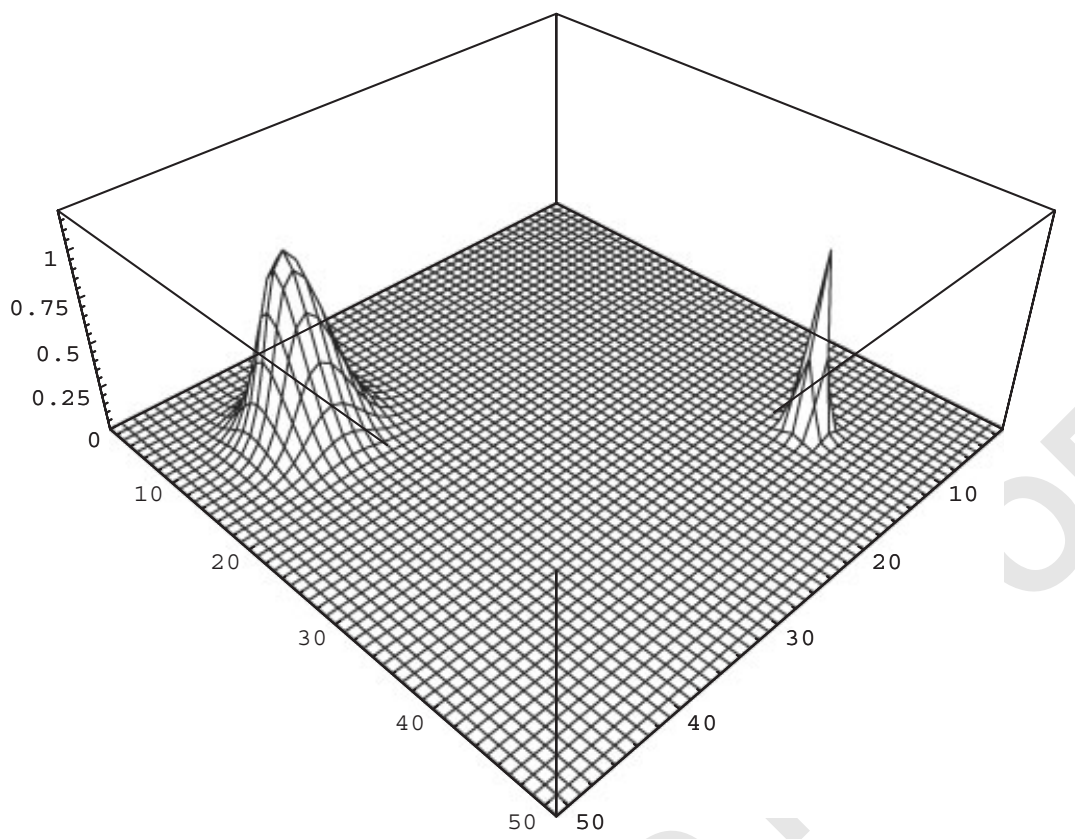

Figure 2. The graphs of 2D delta-shaped functions by Abel's regularization technique with $M=20, \alpha=0.01$ and $M=200, \alpha=0.0003$, respectively.

1 The basis functions of the MAFS for the general linear differential operators such as (7) can be obtained in a similar way.

\section{FINITE-DIFFERENCE TIME-STEPPING ALGORITHM}

As mentioned above, the trigonometric functions $\varphi_{n}(x)$ and their products form a natural basis for

5 the problems we considered. Through this section we consider the following $2 \mathrm{D}$ heat conduction equation:

$$
\frac{\partial u(\mathbf{x}, t)}{\partial t}=\chi \nabla^{2} u(\mathbf{x}, t)+f(\mathbf{x}, t)
$$

where $\chi$ is a constant.

9 Let us recall $\mathrm{C}-\mathrm{N}$ scheme used in the finite-difference approximation of the parabolic equations. In particular, $\mathrm{C}-\mathrm{N}$ scheme for the heat equation in one spatial dimension $\partial_{t} u=\chi \partial_{x x} u$ can be

11 expressed in the form

$$
\frac{u^{n+1}-u^{n}}{\Delta t}=\frac{\chi}{2}\left[D_{x x}\left[u^{n+1}\right]+D_{x x}\left[u^{n}\right]\right]
$$


1 where $D_{x x}$ denotes the finite-difference approximation of the second derivative. In the case $\partial_{t} u=$ $\chi\left(\partial_{x x} u+\partial_{y y} u\right)$ with two spatial dimensions, the $\mathrm{C}-\mathrm{N}$ scheme can be written in a similar form

$$
\frac{u^{n+1}-u^{n}}{\Delta t}=\frac{\chi}{2}\left[D_{x x, y y}\left[u^{n+1}\right]+D_{x x, y y}\left[u^{n}\right]\right]
$$

where $D_{x x, y y}$ denotes the well-known finite-difference approximation of the Laplacian.

To approximate Equation (1), we follow a similar format as above

$$
\frac{u^{n+1}-u^{n}}{\Delta t}=\frac{1}{2}\left[L_{x x, y y}\left[u^{n+1}\right]+L_{x x, y y}\left[u^{n}\right]\right]+f\left(\mathbf{x}, t^{n+1 / 2}\right)
$$

7 which approximates the PDE with second order in time at the time moment $t^{n+1 / 2}$. However, $L_{x x, y y}$ here denotes the spectral approximation instead of the finite-difference approximation for

9 the space operator $L$ in (1). Hence, strictly speaking, we do not use the $\mathrm{C}-\mathrm{N}$ approximation scheme but the likewise $\mathrm{C}-\mathrm{N}$ scheme that has the same second-order approximation in time and the average

11 of the space operators on $u^{n+1}$ and $u^{n}$. When $L=\chi \nabla^{2}$, using the likewise $\mathrm{C}-\mathrm{N}$ scheme, we obtain

$$
\frac{u^{n+1}-u^{n}}{\Delta t}=\frac{\chi}{2}\left(\nabla^{2} u^{n+1}+\nabla^{2} u^{n}\right)+f\left(\mathbf{x}, t^{n+1 / 2}\right)
$$

13 i.e.

$$
\nabla^{2} u^{n+1}-\frac{2}{\chi \Delta t} u^{n+1}=-\nabla^{2} u^{n}-\frac{2}{\chi \Delta t} u^{n}-\frac{2}{\chi} f\left(\mathbf{x}, t^{n+1 / 2}\right)
$$

15 The inhomogeneous Helmholtz equation (26) can be rewritten as

$$
\nabla^{2} u^{n+1}-p u^{n+1}=h^{n}
$$

17 where

$$
p=\frac{2}{\chi \Delta t}, \quad h^{n}=-\nabla^{2} u^{n}-p u^{n}-\frac{2}{\chi} f\left(\mathbf{x}, t^{n+1 / 2}\right)
$$

19 We note that the evaluation of $\nabla^{2} u^{n}$ in (26) at each time step is tedious and it may subject to the loss of accuracy due to the difficulty in evaluating the second derivative. To avoid these difficulties,

21 we modify the above numerical scheme by applying the algorithm proposed by Ramachandran and Balakrishnan [16]. By introducing the intermediate variable

$$
\tilde{u}=\frac{1}{2}\left(u^{n+1}+u^{n}\right)
$$

(26) can be re-casted in the following form:

$$
\frac{2 \widetilde{u}-2 u^{n}}{\Delta t}=\chi \nabla^{2} \widetilde{u}+f\left(\mathbf{x}, t^{n+1 / 2}\right)
$$

or

$$
\nabla^{2} \widetilde{u}-\frac{2}{\chi \Delta t} \widetilde{u}=-\frac{2}{\chi \Delta t} u^{n}-\frac{1}{\chi} f\left(\mathbf{x}, t^{n+1 / 2}\right)
$$

The values of $u^{n+1}$ can then be obtained from (29) after $\tilde{u}$ is computed from (31). We employ both 29 approaches (26) and (31) in the section of numerical results and observe little difference between them. In both cases, we need to evaluate the approximate particular solution. 


\section{THE METHOD OF PARTICULAR SOLUTIONS}

We would like to start this section with a brief review of the two-stage approximation scheme introduced in [5]. Here, we focus on the bivariate case since higher dimensional cases can be dealt with by a similar scheme. Let us consider a function $g(x, y)$. The main idea of this approximation scheme is to perform Chebyshev interpolation to $g(x, y)$ first and then to further approximate the Chebyshev polynomials by the $C$-expansion.

7 The Chebyshev interpolant using Gauss-Lobatto nodes [17-19] for rectangular $[a, b] \times[c, d]$ takes the form

$$
\widetilde{g}(x, y)=\sum_{i=0}^{N_{x}} \sum_{j=0}^{N_{y}} a_{i j} T_{i}\left(\frac{2 x-b-a}{b-a}\right) T_{j}\left(\frac{2 y-d-c}{d-c}\right)
$$

where

$$
a_{i j}=\frac{4}{N_{x} N_{y} \bar{c}_{i} \bar{c}_{j}} \sum_{p=0}^{N_{x}} \sum_{q=0}^{N_{y}} \frac{f\left(x_{p}, y_{q}\right)}{\bar{c}_{p} \bar{c}_{q}} \cos \left(\frac{\pi p i}{N_{x}}\right) \cos \left(\frac{\pi q j}{N_{y}}\right)
$$

and $\bar{c}_{0}=\bar{c}_{N_{x}}=\bar{c}_{N_{y}}=2, \bar{c}_{i}=1,1 \leqslant i \leqslant N_{x}-1$, and $\bar{c}_{j}=1,1 \leqslant j \leqslant N_{y}-1$. Note that $N_{x}$ and $N_{y}$ are the numbers of Gauss-Lobatto nodes in the $x$ and $y$ directions, respectively. It is well known that the use of Gauss-Lobatto nodes will ensure the spectral convergence for the Chebyshev interpolation.

15 The above Chebyshev interpolation is followed by the $C$-expansion procedure. Instead of using the multi-dimensional generalization of the $C$-expansion procedure, we use $1 \mathrm{D} C$-expansion since

17 every term in the right-hand side of (32) is a product of 1D functions. For each 1D function $T_{i}((2 x-b-a) /(b-a))$, its $C$-expansion is in the form

$$
T_{i}\left(\frac{2 x-b-a}{b-a}\right) \simeq \sum_{m=1}^{M} t_{i, m} \varphi_{m}(x)
$$

where $\varphi_{m}$ are given in (9) and $t_{i, m}$ are the expansion coefficients. Thus, the $C$-expansion for $T_{i}((2 x-b-a) /(b-a)) T_{i}((2 y-d-c) /(d-c))$ is given by

$$
T_{i}\left(\frac{2 x-b-a}{b-a}\right) T_{j}\left(\frac{2 y-d-c}{d-c}\right) \simeq \sum_{\mathbf{m}=\mathbf{1}}^{\mathbf{M}} T_{m_{1}, m_{2}}^{i, j} \varphi_{m_{1}}(x) \varphi_{m_{2}}(y)
$$

23 where $\mathbf{m}=\left(m_{1}, m_{2}\right), \mathbf{M}=\left(M_{1}, M_{2}\right), \mathbf{1}=(1,1), T_{m_{1}, m_{2}}^{i, j}=t_{i, m_{1}} t_{j, m_{2}}$.

By combining approximations for all terms $a_{i j} T_{i} T_{j}$ in the right-hand side of (32), the two-stage approximation for the given function $g(x, y)$ gives the following form:

$$
g(x, y) \simeq \sum_{\mathbf{m}=\mathbf{1}}^{\mathbf{M}} G_{m_{1}, m_{2}} \varphi_{m_{1}}(x) \varphi_{m_{2}}(y)
$$

27 where

$$
G_{m_{1}, m_{2}}=\sum_{i=0}^{N_{x}} \sum_{j=0}^{N_{y}} T_{m_{1}, m_{2}}^{i, j}
$$




\section{NME 2230}

1 Hence, by the above two-stage approximation process, $u^{n}$ in (28) can be approximated by the truncated trigonometric series:

$$
u^{n}(\mathbf{x}) \simeq \sum_{\mathbf{m}=\mathbf{1}}^{\mathbf{M}} U_{m_{1}, m_{2}}^{n} \varphi_{m_{1}}\left(x_{1}\right) \varphi_{m_{2}}\left(x_{2}\right)
$$

Similarly, $f\left(\mathbf{x}, t^{n+1 / 2}\right)$ in (28) can be approximated as follows:

$$
f\left(\mathbf{x}, t^{n+1 / 2}\right) \simeq \sum_{\mathbf{m}=\mathbf{1}}^{\mathbf{M}} F_{m_{1}, m_{2}}^{n} \varphi_{m_{1}}\left(x_{1}\right) \varphi_{m_{2}}\left(x_{2}\right)
$$

where the coefficients $U_{m_{1}, m_{2}}^{n}$ and $F_{m_{1}, m_{2}}^{n}$ in (34) and (35) are to be determined by (33). As a

7 result, the right-hand side of (27) can be expressed in the same form

$$
h^{n}(\mathbf{x}) \simeq \sum_{\mathbf{m}=\mathbf{1}}^{\mathbf{M}} H_{m_{1}, m_{2}}^{n} \varphi_{m_{1}}\left(x_{1}\right) \varphi_{m_{2}}\left(x_{2}\right)
$$

9 where

$$
H_{m_{1}, m_{2}}^{n}=\left(\lambda_{m_{1}}^{2}+\lambda_{m_{2}}^{2}-p\right) U_{m_{1}, m_{2}}^{n}-\frac{2}{\chi} F_{m_{1}, m_{2}}^{n}
$$

11 and $\left\{\lambda_{m_{1}}, \lambda_{m_{2}}\right\}$ are given in (9).

13 form:

Using the method of particular solutions [2], we can split the solution of (27) into the following

$$
u^{n+1}=u_{p}^{n+1}+u_{h}^{n+1}
$$

15 where $u_{p}^{n+1}$ is a particular solution that does not necessarily satisfy the boundary condition (2) and $u_{h}^{n+1}$ is the corresponding homogeneous solution. Since we approximate the right-hand side

$17 h^{n}(\mathbf{x})$ of (27) by the truncated series, it is easy to find $u_{p}^{n+1}$ in the analytic form

$$
u_{p}^{n+1}(\mathbf{x})=\sum_{\mathbf{m}=\mathbf{1}}^{\mathbf{M}} U_{p, m_{1}, m_{2}}^{n+1} \varphi_{m_{1}}\left(x_{1}\right) \varphi_{m_{2}}\left(x_{2}\right)
$$

19 where

$$
U_{p, m_{1}, m_{2}}^{n+1}=-\frac{H_{m_{1}, m_{2}}^{n}}{\lambda_{m_{1}}^{2}+\lambda_{m_{2}}^{2}+p}
$$

21 The homogeneous solution $u_{h}^{n+1}$ satisfies the corresponding homogeneous Helmholtz equation

$$
\begin{aligned}
\nabla^{2} u_{h}^{n+1}-p u_{h}^{n+1} & =0, \quad \mathbf{x} \in \Omega \\
B\left[u_{h}^{n+1}(\mathbf{x})\right] & =g^{n+1}(\mathbf{x})-B\left[u_{p}^{n+1}(\mathbf{x})\right], \quad \mathbf{x} \in \partial \Omega
\end{aligned}
$$

where $g^{n+1}(\mathbf{x})=g\left(\mathbf{x}, t^{n+1}\right)$. Note that $u_{h}^{n+1}$ can be approximated by the linear combination of the 23 approximate fundamental solutions $\Psi\left(\mathbf{x}, \boldsymbol{\xi}_{k}\right)$ in (22), i.e.

$$
u_{h}^{n+1} \simeq \sum_{k=1}^{K} q_{k} \Psi\left(\mathbf{x}, \xi_{k}\right)
$$


1 where $q_{k}$ are coefficients to be determined and the source points $\xi_{k}$ are placed outside the solution domain $\Omega$. By fitting the boundary condition in (41) using collocation method, $q_{k}$ can be easily

3 determined [2]. It is interesting that the particular solution and the fundamental solution have the same basis functions. After $q_{k}$ are obtained, the solution at each time step can be obtained as

5 follows:

$$
u^{n+1}(\mathbf{x}) \simeq \sum_{\mathbf{m}=\mathbf{1}}^{\mathbf{M}} U_{m_{1}, m_{2}}^{n+1} \varphi_{m_{1}}\left(x_{1}\right) \varphi_{m_{2}}\left(x_{2}\right)
$$

7

where

$$
\begin{aligned}
U_{m_{1}, m_{2}}^{n+1} & =U_{p, m_{1}, m_{2}}^{n+1}+\sum_{k=1}^{K} q_{k}^{n+1} D_{m_{1}, m_{2}}^{k} \\
D_{m_{1}, m_{2}}^{k}(\xi, \eta) & =-\frac{c_{m_{1}, m_{2}}\left(\xi_{k}, \eta_{k}\right)}{\lambda_{m_{1}}^{2}+\lambda_{m_{2}}^{2}+p}
\end{aligned}
$$

9 Starting from the initial condition by the two-stage interpolation scheme, we carry out the proposed calculations (34)-(45) at each time step.

\section{NUMERICAL RESULTS}

\section{1. $2 D$ cases}

13 From our numerical experiments, the Riesz regularization technique (14) leads to the divergence of the solution. Hence, throughout this section we use Lanczos and Abel regularizing techniques

15 only. In all the calculations presented in this section the source points are distributed on a fictitious boundary which is a circle with its center at $(0,0)$ and radius $R_{S}=0.99$. The number of the

17 collocation points on $\partial \Omega$ is taken as twice as many as that of the source points. We test our algorithm on problems with known exact solutions $u_{\mathrm{ex}}$. We also compare some of the problems with the

19 traditional finite difference method and meshless method in [20]. To validate the performance of the proposed algorithm, the mean square root (MSR) errors

$$
\operatorname{MSR}=\sqrt{\frac{1}{N_{e}} \sum_{i=1}^{N_{e}}\left[u\left(x_{i}, y_{i}, t\right)-u_{\mathrm{ex}}\left(x_{i}, y_{i}, t\right)\right]^{2}}
$$

are computed using a uniform $9 \times 9$ mesh for the square domain, where $N_{e}$ is the total number 23 of nodes on the mesh. In the case of irregular domain the test points are obtained using RNUF pseudorandom number generator from the Microsoft IMSL Library.

25 Since approximations (34)-(36) vanish on the boundary of the square $[-1,1] \times[-1,1]$ due to the zero boundary condition (10) of $\varphi_{n}(x)$, in the following we consider problems defined either

27 on the square $[-0.5,0.5] \times[-0.5,0.5]$ or in an irregular region that is strictly inside the square $[-0.5,0.5] \times[-0.5,0.5]$ and bounded away from $[-1,1] \times[-1,1]$. If this is not the case originally,

29 appropriate translation and scaling operations are required. In the following examples, the numbers of Chebyshev's polynomials for $x$ and $y$ directions are denoted, respectively, by $N_{x}$ and $N_{y}$, the 
1 number of trigonometric harmonics in the $C$-expansion is denoted by $M$, and the number of source points for the MAFS is denoted by $K$.

\section{Example 1}

Let us consider the following diffusion equation:

$$
\frac{\partial u(x, y, t)}{\partial t}=\nabla^{2} u(x, y, t)+f(x, y, t), \quad(x, y) \in \Omega, \quad t>0
$$

where $\Omega=[-0.5,0.5] \times[-0.5,0.5]$. The initial condition, the Dirichlet boundary condition, and

$7 f(x, y, t)$ are chosen such that the exact solution is

$$
u_{\mathrm{ex}}(x, y, t)=\sin [(x+0.5)(y+0.5)] \cos t
$$

9 The numerical results with Lanczos regularization scheme for different time steps $\Delta t$ are shown in Table I. The numerical results in the first four columns are obtained using $N_{x}=N_{y}=25, M=$

$1130, K=30$. Initially, the errors improved when $\Delta t$ decreased. This is consistent with the wellknown $\mathrm{C}-\mathrm{N}$ scheme. This means that the error in the approximation of the PDE was dominated

13 by these parameters. However, for $\Delta t \leqslant \frac{1}{16}$ the error does not improve with the further reduction of $\Delta t$. This implies that the error in the approximation of the PDE becomes the non-dominating

15 one and the error in solution is caused by other reasons. To further reduce the error, we need to improve the approximation of the forcing term using Chebyshev and $C$-expansion scheme [5]. The

17 results in the last column of Table I are obtained using $N_{x}=N_{y}=30, M=50$, and $K=50$.

The same problem is solved using the Abel regularization. We choose the same parameters

$19 N_{x}, N_{y}, M$ and $K$ as in Table I. Some results are presented in Table II. The numerical results in the first four columns are obtained using $\alpha=0.005$ with $M=30$ and in the last column using $\alpha=0.002$ with $M=70$.

Table I. The MSR errors in Example 1 using the Lanczos regularization in a squared domain.

\begin{tabular}{rccccc}
\hline$t$ & $\Delta t=\frac{1}{4}$ & $\Delta t=\frac{1}{8}$ & $\Delta t=\frac{1}{16}$ & $\Delta t=\frac{1}{32}$ & $\Delta t=\frac{1}{32}$ \\
\hline 1 & $1.8 \times 10^{-5}$ & $2.1 \times 10^{-5}$ & $5.9 \times 10^{-6}$ & $7.6 \times 10^{-6}$ & $3.6 \times 10^{-7}$ \\
2 & $6.3 \times 10^{-5}$ & $1.8 \times 10^{-5}$ & $8.0 \times 10^{-6}$ & $8.7 \times 10^{-6}$ & $8.8 \times 10^{-7}$ \\
5 & $5.5 \times 10^{-5}$ & $1.4 \times 10^{-5}$ & $5.7 \times 10^{-6}$ & $6.8 \times 10^{-6}$ & $7.4 \times 10^{-7}$ \\
10 & $5.3 \times 10^{-5}$ & $2.3 \times 10^{-5}$ & $1.7 \times 10^{-5}$ & $1.0 \times 10^{-5}$ & $7.8 \times 10^{-7}$ \\
\hline
\end{tabular}

Table II. The MSR errors and CPU time in Example 1 using the Abel regularization in a squared domain.

\begin{tabular}{cccccc}
\hline$t$ & $\Delta t=\frac{1}{4}$ & $\Delta t=\frac{1}{8}$ & $\Delta t=\frac{1}{16}$ & $\Delta t=\frac{1}{32}$ & $\Delta t=\frac{1}{32}$ \\
\hline 1 & $1.8 \times 10^{-5}$ & $9.8 \times 10^{-6}$ & $2.4 \times 10^{-6}$ & $7.6 \times 10^{-6}$ & $3.6 \times 10^{-7}$ \\
2 & $6.3 \times 10^{-5}$ & $1.6 \times 10^{-5}$ & $4.1 \times 10^{-6}$ & $8.7 \times 10^{-6}$ & $8.8 \times 10^{-7}$ \\
5 & $5.5 \times 10^{-5}$ & $1.2 \times 10^{-5}$ & $3.6 \times 10^{-6}$ & $6.8 \times 10^{-6}$ & $7.4 \times 10^{-7}$ \\
10 & $5.3 \times 10^{-5}$ & $1.5 \times 10^{-5}$ & $6.5 \times 10^{-6}$ & $1.0 \times 10^{-5}$ & $7.8 \times 10^{-7}$ \\
CPU & 7.4 & 14.3 & 28.0 & 55.6 & 137.0 \\
\hline & & & & &
\end{tabular}


Table III. The MSR errors and CPU time in Example 1 using C-N approximation scheme of the FDM in a squared domain.

\begin{tabular}{cccccc}
\hline$t$ & $\Delta t=\frac{1}{4}$ & $\Delta t=\frac{1}{8}$ & $\Delta t=\frac{1}{16}$ & $\Delta t=\frac{1}{32}$ & $\Delta t=\frac{1}{32}$ \\
\hline 1 & $1.3 \times 10^{-5}$ & $4.6 \times 10^{-6}$ & $1.6 \times 10^{-6}$ & $8.0 \times 10^{-7}$ & $4.0 \times 10^{-7}$ \\
2 & $5.0 \times 10^{-5}$ & $1.2 \times 10^{-5}$ & $3.3 \times 10^{-6}$ & $1.1 \times 10^{-6}$ & $8.6 \times 10^{-7}$ \\
5 & $4.1 \times 10^{-5}$ & $1.0 \times 10^{-5}$ & $2.7 \times 10^{-6}$ & $8.5 \times 10^{-7}$ & $7.0 \times 10^{-7}$ \\
10 & $4.4 \times 10^{-5}$ & $1.3 \times 10^{-5}$ & $3.5 \times 10^{-6}$ & $1.5 \times 10^{-6}$ & $8.9 \times 10^{-7}$ \\
CPU & 0.31 & 0.64 & 1.22 & 2.43 & 36.35 \\
\hline
\end{tabular}

Table IV. The MSR errors and CPU time in Example 1 using the Abel regularization in a squared domain.

\begin{tabular}{cccccc}
\hline$t$ & $\Delta t=\frac{1}{4}$ & $\Delta t=\frac{1}{8}$ & $\Delta t=\frac{1}{16}$ & $\Delta t=\frac{1}{32}$ & $\Delta t=\frac{1}{32}$ \\
\hline 1 & $1.4 \times 10^{-3}$ & $4.6 \times 10^{-4}$ & $2.3 \times 10^{-4}$ & $1.1 \times 10^{-4}$ & $4.8 \times 10^{-5}$ \\
2 & $5.4 \times 10^{-3}$ & $8.1 \times 10^{-4}$ & $1.7 \times 10^{-4}$ & $7.9 \times 10^{-5}$ & $3.6 \times 10^{-5}$ \\
5 & $1.0 \times 10^{-3}$ & $3.8 \times 10^{-4}$ & $1.1 \times 10^{-4}$ & $5.5 \times 10^{-5}$ & $2.5 \times 10^{-5}$ \\
10 & $5.4 \times 10^{-3}$ & $1.2 \times 10^{-3}$ & $3.5 \times 10^{-4}$ & $1.8 \times 10^{-4}$ & $7.5 \times 10^{-5}$ \\
CPU & 19.1 & 35.9 & 69.7 & 137.3 & 170.2 \\
\hline
\end{tabular}

Table V. The MSR errors and CPU time in Example 1 using C-N approximation scheme of the FDM in a squared domain.

\begin{tabular}{ccccc}
\hline$t$ & $21 \times 21$ mesh & $41 \times 41$ mesh & $61 \times 61$ mesh & $81 \times 81 \mathrm{mesh}$ \\
\hline 1 & $1.3 \times 10^{-2}$ & $3.5 \times 10^{-3}$ & $1.6 \times 10^{-3}$ & $9.1 \times 10^{-4}$ \\
2 & $1.1 \times 10^{-2}$ & $2.7 \times 10^{-3}$ & $1.2 \times 10^{-3}$ & $6.9 \times 10^{-4}$ \\
5 & $7.1 \times 10^{-3}$ & $1.8 \times 10^{-3}$ & $8.2 \times 10^{-4}$ & $4.7 \times 10^{-4}$ \\
10 & $2.1 \times 10^{-2}$ & $5.4 \times 10^{-3}$ & $2.5 \times 10^{-3}$ & $1.4 \times 10^{-3}$ \\
$\mathrm{CPU}$ & 2.1 & 28.5 & 152.0 & 450.0 \\
\hline
\end{tabular}

1 We compare our method with the classical finite difference method (FDM) using C-N scheme for this problem on $\Omega=[0,1] \times[0,1]$ in which the corresponding exact solution is $u_{\mathrm{ex}}=\sin (x y) \cos (t)$.

3 The numerical results in the first four columns of Table III are obtained using $21 \times 21$ uniform mesh while the results in the last column are obtained using $41 \times 41$ uniform mesh. By comparing

5 the results shown in Tables II and III, it is evident that the FDM is more efficient than our method. However, the situation changes when we consider the problem with more complex exact solution

7 which has more oscillations:

$$
u_{\mathrm{ex}}(x, y, t)=\sin (20 x y) \cos (t)
$$

9 on $\Omega=[0,1] \times[0,1]$ by the FDM, which corresponds to the exact solution

$$
u_{\mathrm{ex}}(x, y, t)=\sin [20(x+0.5)(y+0.5)] \cos (t)
$$




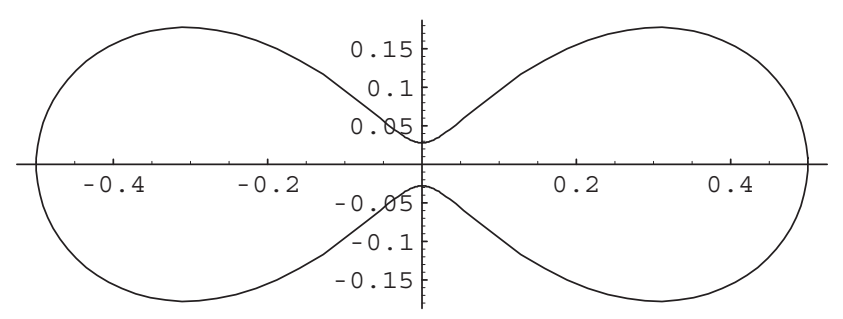

Figure 3. Oval of Cassini.

Table VI. The MSR errors in Example 2 using the Lanczos regularization in the domain of oval of Cassini.

\begin{tabular}{rcccc}
\hline$t$ & $\Delta t=\frac{1}{4}$ & $\Delta t=\frac{1}{8}$ & $\Delta t=\frac{1}{16}$ & $\Delta t=\frac{1}{16}$ \\
\hline 1 & $4.6 \times 10^{-6}$ & $2.8 \times 10^{-7}$ & $2.2 \times 10^{-7}$ & $2.5 \times 10^{-7}$ \\
2 & $1.3 \times 10^{-5}$ & $2.2 \times 10^{-6}$ & $5.3 \times 10^{-7}$ & $5.6 \times 10^{-7}$ \\
5 & $5.7 \times 10^{-6}$ & $1.7 \times 10^{-6}$ & $4.5 \times 10^{-7}$ & $4.3 \times 10^{-7}$ \\
10 & $7.2 \times 10^{-6}$ & $1.8 \times 10^{-6}$ & $3.9 \times 10^{-6}$ & $4.3 \times 10^{-7}$ \\
\hline
\end{tabular}

1 on $\Omega=[-0.5,0.5] \times[-0.5,0.5]$ by our method. The computational results are shown in Tables IV and V. We observe that our proposed method is better than FDM. The results shown in the

3 first four columns of Table IV are obtained using $N_{x}=N_{y}=30, M=70, K=50, \alpha=0.002$, and the results shown in the last column correspond to $N_{x}=N_{y}=30, M=100, K=50, \alpha=0.0012$. In

5 Table $\mathrm{V}$, the results are obtained using FDM with $\Delta t=\frac{1}{32}$ and the best results are obtained using $81 \times 81$ mesh which can be easily achieved using our proposed method with fairly large time step

$7 \Delta t=\frac{1}{8}$ as shown in the second column of Table IV. Furthermore, the CPU times are 450 and $35.9 \mathrm{~s}$ for FDM and our proposed method, respectively. The CPU time by FDM increases rapidly with

9 the growth of the mesh size. Hence, we conclude that our proposed method is more effective than FDM for the cases of complicated solution. For a regular domain such as square, FDM may have

11 the advantage for solving smooth problems. However, for irregular domains or high-dimensional problems, our proposed meshless approach has the clear advantage due to the difficulty of meshing

13 the domain using FDM.

\section{Example 2}

15 In this example, we consider the same problem as in the previous example for an irregular-shaped domain as depicted in Figure 3 which is represented by the following parametric equation:

$$
x(t)=R(t) \cos (t), \quad y(t)=R(t) \sin (t)
$$

where

$$
R(t)=c^{2} \cos (2 t)+\sqrt{a^{4}-c^{4} \sin ^{2}(2 t)}, \quad 0 \leqslant t \leqslant 2 \pi
$$

Here we set $c=0.353, a=\sqrt{0.25-c^{2}}$. The numerical results in Table VI are obtained using 21 Lanczos regularization scheme with $N_{x}=N_{y}=25, M=30, K=30$ in the first three columns of the table and $N_{x}=N_{y}=30, M=50, K=30$ in the last column. 
Table VII. The MSR errors in Example 2 using the Abel regularization in the domain of oval of Cassini.

\begin{tabular}{rcccc}
\hline$t$ & $\Delta t=\frac{1}{4}$ & $\Delta t=\frac{1}{8}$ & $\Delta t=\frac{1}{16}$ & $\Delta t=\frac{1}{32}$ \\
\hline 1 & $4.7 \times 10^{-6}$ & $4.2 \times 10^{-7}$ & $3.2 \times 10^{-7}$ & $8.3 \times 10^{-8}$ \\
2 & $1.3 \times 10^{-5}$ & $2.2 \times 10^{-6}$ & $5.8 \times 10^{-7}$ & $1.8 \times 10^{-7}$ \\
5 & $5.7 \times 10^{-6}$ & $1.8 \times 10^{-6}$ & $4.5 \times 10^{-7}$ & $1.6 \times 10^{-7}$ \\
10 & $7.4 \times 10^{-6}$ & $5.5 \times 10^{-6}$ & $5.4 \times 10^{-7}$ & $1.3 \times 10^{-6}$ \\
\hline
\end{tabular}

1 Similar to the last example, the numerical results obtained using Abel's regularization technique are shown in Table VII. The results in the first three columns are obtained using $M=30$ and $K=20$.

3 The results in the last column correspond to $M=50$ and $K=25$. The regularization parameter is taken as $\alpha=0.005$ in all the calculations placed in this table. The collocation points are distributed

5 uniformly, in terms of angle, on the boundary.

For the irregular-shaped domain, it is a major task to meshing the domain using FDM. Our

7 proposed method is meshless and the solution procedure for the previous example with square domain and the current one with irregular domain is the same.

9 To further demonstrate the effectiveness of the proposed algorithm, in the following we show two examples with more complicated forcing term.

11 Example 3

Let us consider the problem with the same diffusion equation (46) in the domain $\Omega=[-0.5,0.5] \times$

$13[-0.5,0.5]$ with the initial and Dirichlet boundary conditions and $f(x, y, t)$ being artificially imposed in such a way that the exact solution is

$$
u_{\mathrm{ex}}(x, y, t)=f_{1}(x, y) \cos t
$$

where

$$
\begin{aligned}
f_{1}(x, y)= & \frac{3}{4} \exp \left(-\frac{(9 x+2.5)^{2}+(9 y+2.5)^{2}}{4}\right)+\frac{3}{4} \exp \left(-\frac{(9 x+5.5)^{2}+(9 y+5.5)^{2}}{49}\right) \\
& +\frac{1}{2} \exp \left(-\frac{(9 x-2.5)^{2}+(9 y+1.5)^{2}}{4}\right)-\frac{1}{5} \exp \left(-(9 x+0.5)^{2}-(9 y-2.5)^{2}\right)
\end{aligned}
$$

17 Note that $f_{1}(x, y)$ is a re-scaled Franke's function (see Figure 4) which is widely used as a benchmark problem for surface reconstruction [21]. The function $f_{1}(x, y)$ in (48) was originally

19 defined in the unit square.

To obtain the numerical results, we couple the Chebyshev and $C$-expansion approximation with

21 the Lanczos regularization scheme and let $N_{x}=N_{y}=45, M=100, K=100$. The numerical results with different time steps $\Delta t$ are shown in Table VIII.

23 The numerical results shown in Table IX are obtained using Abel's regularization scheme with $\alpha=0.0012, M=100$, and $K=100$. As we can see, the numerical results in both approaches are 25 excellent. 


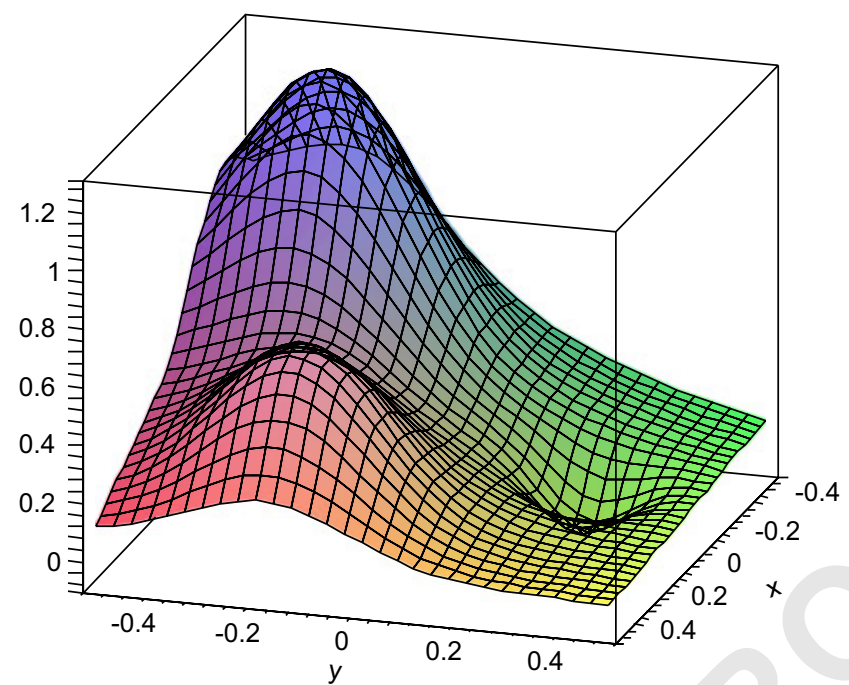

Figure 4. Franke's function $f_{1}(x, y)$.

Table VIII. The MSR errors in Example 3 using the Lanczos regularization in the squared domain.

\begin{tabular}{rccc}
\hline$t$ & $\Delta t=\frac{1}{8}$ & $\Delta t=\frac{1}{16}$ & $\Delta t=\frac{1}{32}$ \\
\hline 1 & $1.6 \times 10^{-4}$ & $4.3 \times 10^{-5}$ & $1.1 \times 10^{-5}$ \\
2 & $1.4 \times 10^{-4}$ & $3.3 \times 10^{-5}$ & $8.2 \times 10^{-6}$ \\
5 & $9.0 \times 10^{-5}$ & $2.2 \times 10^{-5}$ & $6.4 \times 10^{-6}$ \\
10 & $2.7 \times 10^{-4}$ & $6.7 \times 10^{-5}$ & $3.0 \times 10^{-4}$ \\
\hline
\end{tabular}

Table IX. The MSR errors in Example 3 using the Abel regularization in the squared domain.

\begin{tabular}{rccc}
\hline$t$ & $\Delta t=\frac{1}{8}$ & $\Delta t=\frac{1}{16}$ & $\Delta t=\frac{1}{32}$ \\
\hline 1 & $1.6 \times 10^{-4}$ & $4.5 \times 10^{-5}$ & $1.4 \times 10^{-5}$ \\
2 & $1.4 \times 10^{-4}$ & $3.4 \times 10^{-5}$ & $8.6 \times 10^{-6}$ \\
5 & $9.0 \times 10^{-5}$ & $2.3 \times 10^{-5}$ & $2.0 \times 10^{-6}$ \\
10 & $2.7 \times 10^{-4}$ & $6.7 \times 10^{-5}$ & $4.5 \times 10^{-4}$ \\
\hline
\end{tabular}

1 Example 4

In this example we consider the same diffusion equation (46) in the domain $\Omega=[-0.5,0.5] \times$

$3[-0.5,0.5]$. The initial condition, the Dirichlet boundary condition, and $f(x, y, t)$ are imposed such that the exact solution is

$$
u_{\mathrm{ex}}(x, y, t)=f_{2}(x, y) \cos t
$$




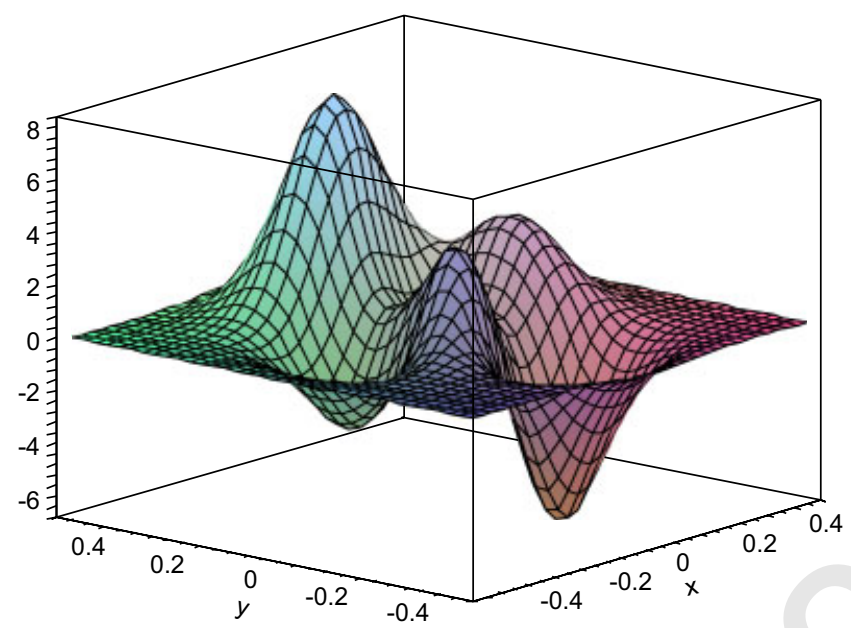

Figure 5. The PEAK's function $f_{2}(x, y)$.

Table X. The MSR errors in Example 4 using the Lanczos regularization in the squared domain.

\begin{tabular}{rccc}
\hline$t$ & $\Delta t=\frac{1}{8}$ & $\Delta t=\frac{1}{16}$ & $\Delta t=\frac{1}{32}$ \\
\hline 1 & $1.8 \times 10^{-3}$ & $5.5 \times 10^{-4}$ & $1.5 \times 10^{-4}$ \\
2 & $2.0 \times 10^{-3}$ & $4.2 \times 10^{-4}$ & $1.1 \times 10^{-4}$ \\
5 & $1.1 \times 10^{-3}$ & $2.8 \times 10^{-4}$ & $7.2 \times 10^{-4}$ \\
10 & $3.5 \times 10^{-3}$ & $8.7 \times 10^{-4}$ & $1.6 \times 10^{-3}$ \\
\hline
\end{tabular}

where

$$
\begin{aligned}
f_{2}(x, y)= & 3(1-6 x)^{2} \exp \left(-36 x^{2}-(6 y+1)^{2}\right)-10\left(\frac{6 x}{5}-(6 x)^{3}-(6 y)^{5}\right) \\
& \times \exp \left(-36\left(x^{2}+y^{2}\right)\right)-\frac{1}{3} \exp \left(-(6 x+1)^{2}-36 y^{2}\right)
\end{aligned}
$$

is the re-scaled PEAKS function (see Figure 5) from MATLAB [22]. To evaluate the particular solutions, we use the Chebyshev and $C$-expansion approximation with Lanczos regularization scheme and let $N_{x}=N_{y}=40, M=100$, and $K=100$. The numerical results with different time

5 steps $\Delta t$ are shown in Table $\mathrm{X}$. Using Abel's regularization scheme with the parameters $\alpha=$ $0.0012, M=100$, and $K=100$, we obtain the numerical results as shown in Table XI.

7 We note that the FDM scheme cannot give any reasonable solution for the problems in Examples 3 and 4.

9 The previous examples have shown the effectiveness of our proposed method in solving problems with complicated domain or forcing term. In the next example, we will demonstrate how our

11 method can be applied to problems with different types of boundary conditions. By examining 
Table XI. The MSR errors in Example 4 using the Abel regularization in the squared domain.

\begin{tabular}{rccc}
\hline \multicolumn{1}{c}{$t$} & $\Delta t=\frac{1}{8}$ & $\Delta t=\frac{1}{16}$ & $\Delta t=\frac{1}{32}$ \\
\hline 1 & $1.8 \times 10^{-3}$ & $5.5 \times 10^{-4}$ & $1.4 \times 10^{-4}$ \\
2 & $2.0 \times 10^{-3}$ & $4.2 \times 10^{-4}$ & $1.1 \times 10^{-4}$ \\
5 & $1.1 \times 10^{-3}$ & $2.8 \times 10^{-4}$ & $7.1 \times 10^{-5}$ \\
10 & $3.5 \times 10^{-3}$ & $8.8 \times 10^{-4}$ & $1.2 \times 10^{-3}$ \\
\hline
\end{tabular}

Table XII. The MSR errors in Example (5) using the Abel regularization in a squared domain with mixed boundary conditions.

\begin{tabular}{rccccc}
\hline$t$ & $\Delta t=\frac{1}{4}$ & $\Delta t=\frac{1}{8}$ & $\Delta t=\frac{1}{16}$ & $\Delta t=\frac{1}{32}$ & $\Delta t=\frac{1}{32}$ \\
\hline 1 & $9.4 \times 10^{-5}$ & $3.8 \times 10^{-5}$ & $4.9 \times 10^{-5}$ & $8.2 \times 10^{-6}$ & $1.8 \times 10^{-6}$ \\
2 & $1.3 \times 10^{-4}$ & $7.0 \times 10^{-5}$ & $2.3 \times 10^{-5}$ & $9.3 \times 10^{-6}$ & $6.1 \times 10^{-6}$ \\
5 & $1.2 \times 10^{-4}$ & $4.0 \times 10^{-5}$ & $7.2 \times 10^{-6}$ & $8.3 \times 10^{-6}$ & $4.7 \times 10^{-6}$ \\
10 & $1.3 \times 10^{-4}$ & $3.1 \times 10^{-5}$ & $1.1 \times 10^{-5}$ & $1.8 \times 10^{-5}$ & $6.6 \times 10^{-6}$ \\
\hline
\end{tabular}

1 Equations (40)-(42), we obtain a collocation system with the matrix $B\left[\Psi\left(\mathbf{x}_{i}, \boldsymbol{\xi}_{k}\right)\right]$, i.e. the entries of the collocation matrix are obtained by applying the boundary operator $B$ to the approximate

3 fundamental solutions $\Psi\left(\mathbf{x}, \xi_{k}\right)$, and then evaluating at the boundary collocation points $\mathbf{x}_{i}$. For example, the entries of the collocation matrix corresponding to Neumann boundary condition is $5 \quad \partial \Psi\left(\mathbf{x}_{i}, \xi_{k}\right) / \partial n$.

\section{Example 5}

7 We consider the same diffusion equation in Example 1 with mixed boundary conditions in the domain $\Omega=[-0.5,0.5] \times[-0.5,0.5]$. The mixed boundary conditions are

$$
\begin{aligned}
u(-0.5, y, t) & =g_{1}(y, t), & -0.5 \leqslant y \leqslant 0.5, & t>0 \\
u(0.5, y, t) & =g_{2}(y, t), & & -0.5 \leqslant y \leqslant 0.5, \quad t>0 \\
u(x,-0.5, t)+\frac{\partial u}{\partial n}(x,-0.5, t) & =g_{3}(x, t), & & -0.5 \leqslant x \leqslant 0.5, \quad t>0 \\
u(x, 0.5, t)+\frac{\partial u}{\partial n}(x, 0.5, t) & =g_{4}(x, t), & & -0.5 \leqslant x \leqslant 0.5, \quad t>0
\end{aligned}
$$

9 The boundary data $g_{i}, i=1,2,3,4$, the source function $f(x, y, t)$, and the initial condition $u(x, y, 0)$ are given according to the exact solution $u_{\mathrm{ex}}(x, y, t)=\sin [(x+0.5)(y+0.5)] \cos t$. The results

11 using Abel regularization with various $\Delta t$ are given in Table XII. The results in the first four columns are obtained using $N_{x}=N_{y}=25, M=50, K=30, \alpha=0.003$ and in the last column using $13 N_{x}=N_{y}=25, M=50, K=50, \alpha=0.003$. Again, the results in both tables are extremely accurate. 


\section{Example 6}

We consider the following diffusion equation:

$$
\frac{\partial u}{\partial t}=\chi\left(\frac{\partial^{2} u}{\partial x^{2}}+\frac{\partial^{2} u}{\partial y^{2}}\right), \quad(x, y) \in D, \quad t>0
$$

with initial and boundary conditions

$$
\begin{aligned}
& u(x, y, 0)=1, \quad(x, y) \in D \\
& u(x, y, t)=0, \quad(x, y) \in \partial D, \quad t>0
\end{aligned}
$$

5 where $D=[-0.2,0.2] \times[-0.2,0.2]$, and $\chi=5.8 \times 10^{-7}$. Note that the solution is not continuous at $t=0$.

7 To apply our method, we need to transform the domain $D$ to the standard domain $\Omega=$ $[-0.5,0.5] \times[-0.5,0.5]$. After the domain transformation, we have

$$
\begin{aligned}
\frac{\partial u}{\partial t} & =\frac{\chi}{0.16}\left(\frac{\partial^{2} u}{\partial x^{2}}+\frac{\partial^{2} u}{\partial y^{2}}\right), \quad(x, y) \in \Omega, \quad t>0 \\
u(x, y, 0) & =1, \quad(x, y) \in \Omega \\
u(x, y, t) & =0, \quad(x, y) \in \partial \Omega, \quad t>0
\end{aligned}
$$

The exact solution is given by [23] as follows:

$$
u_{\mathrm{ex}}(x, y, t)=\frac{16}{\pi^{2}} \sum_{n=0}^{\infty} \sum_{m=0}^{\infty} L_{n, m} \cos [(2 n+1) \pi x] \cos [(2 m+1) \pi y] \exp \left(-D_{n, m} t\right)
$$

where

$$
L_{n, m}=\frac{(-1)^{n+m}}{(2 n+1)(2 m+1)} \quad \text { and } \quad D_{n, m}=\frac{\chi}{0.16} \frac{\pi^{2}}{4}\left[\frac{(2 n+1)^{2}}{0.25}+\frac{(2 m+1)^{2}}{0.25}\right]
$$

In Table XIII, we present some numerical results using Lanczos regularization technique. In this table, the results in the last column are obtained using (31). It should be noted that the calculations are performed with very large values of the parameter $p$ as shown in (28). Note that $\chi$ in (28)

17 should be replaced by $\chi / 0.16$; i.e. $p=0.32 /(\chi \Delta t)$. Thus, $p=110344$ for $\Delta t=5$ and $p=551724$ for $\Delta t=1$. In all the calculations in this example, the number of Chebyshev's polynomials in

19 each axis direction is $N_{x}=N_{y}=5$; the number of the sources is $K=50$. The numerical results in Table XIV are obtained using Abel's regularization with $\alpha=0.0055, M=50$.

\section{Example 7}

In this example we consider the problem given by Šarler and Vertnik [20]; i.e. the diffusion equation (51) in $D=[0,1] \times[0,1]$ with $\chi=1$, and initial and boundary conditions

$$
\begin{array}{ll}
u(x, y, 0)=1, & (x, y) \in D \\
u(x, 1, t)=0, & 0 \leqslant x \leqslant 1, \quad t>0 \\
u(1, y, t)=0, & 0 \leqslant y \leqslant 1, \quad t>0
\end{array}
$$


Table XIII. The MSR errors in Example 6 using the Lanczos regularization.

\begin{tabular}{|c|c|c|c|c|c|}
\hline \multirow[b]{2}{*}{$t$} & \multicolumn{3}{|c|}{$\Delta t=5$} & \multicolumn{2}{|r|}{$\Delta t=1$} \\
\hline & $M=30$ & $M=40$ & $M=50$ & $M=50$ & $M=50$, Reference $[16]$ \\
\hline 1000 & $3.0 \times 10^{-3}$ & $1.1 \times 10^{-3}$ & $4.3 \times 10^{-4}$ & $3.2 \times 10^{-4}$ & $3.3 \times 10^{-4}$ \\
\hline 2000 & $1.0 \times 10^{-3}$ & $3.3 \times 10^{-4}$ & $2.7 \times 10^{-4}$ & $6.4 \times 10^{-5}$ & $4.1 \times 10^{-5}$ \\
\hline 5000 & $2.3 \times 10^{-4}$ & $1.7 \times 10^{-4}$ & $1.4 \times 10^{-4}$ & $4.7 \times 10^{-5}$ & $2.0 \times 10^{-5}$ \\
\hline 10000 & $1.3 \times 10^{-4}$ & $9.8 \times 10^{-5}$ & $8.3 \times 10^{-5}$ & $2.8 \times 10^{-5}$ & $1.2 \times 10^{-5}$ \\
\hline
\end{tabular}

Table XIV. The MSR errors in Example 6 using the Abel regularization.

\begin{tabular}{rccc}
\hline$t$ & $\Delta t=5, M=50$ & $\Delta t=1, M=50$ & $\Delta t=0.5, M=50$ \\
\hline 1000 & $7.8 \times 10^{-3}$ & $7.8 \times 10^{-3}$ & $7.8 \times 10^{-3}$ \\
2000 & $4.6 \times 10^{-3}$ & $4.6 \times 10^{-3}$ & $4.6 \times 10^{-3}$ \\
5000 & $7.7 \times 10^{-4}$ & $7.7 \times 10^{-4}$ & $7.6 \times 10^{-4}$ \\
10000 & $7.1 \times 10^{-5}$ & $3.7 \times 10^{-5}$ & $3.0 \times 10^{-5}$ \\
\hline
\end{tabular}

$$
\begin{array}{ll}
\frac{\partial}{\partial x} u(0, y, t)=0, & 0 \leqslant y \leqslant 1, \quad t>0 \\
\frac{\partial}{\partial y} u(x, 0, t)=0, & 0 \leqslant x \leqslant 1, \quad t>0
\end{array}
$$

1 The analytical solution [23] of the above problem is given by

$$
u_{\mathrm{ex}}(x, y, t)=W(x, t) W(y, t)
$$

3 where

$$
W(x, t)=\frac{4}{\pi} \sum_{n=1}^{\infty} \frac{(-1)^{n+1}}{(2 n-1)} \cos \left(\frac{2 n-1}{2} \pi x\right) \exp \left[-\left(\frac{2 n-1}{2} \pi\right)^{2} t\right]
$$

5 We would like to indicate that there is a misprint for $W(x, t)$ in Equation (35) in [20].

We apply the algorithm described in Example 6 using the Lanczos regularization. The numerical 7 results obtained in Table XV use the following parameters: $N_{x}=N_{y}=5, M=50, K=50, \Delta t=$ $10^{-5}$. To compare our results with the results in Reference [20], we define the average absolute

9 error $\left\|u-u_{\mathrm{ex}}\right\|_{\mathrm{avg}}$ and the maximal absolute error $\left\|u-u_{\mathrm{ex}}\right\|_{\infty}$ as in Reference [20] which are

$$
\begin{aligned}
\left\|u-u_{\mathrm{ex}}\right\|_{\infty} & =\max _{1 \leqslant i \leqslant N_{t}}\left|u\left(t, x_{i}, y_{i}\right)-u_{\mathrm{ex}}\left(t, x_{i}, y_{i}\right)\right| \\
\left\|u-u_{\mathrm{ex}}\right\|_{\mathrm{avg}} & =\frac{1}{N_{t}} \sum_{i=1}^{N_{t}}\left|u\left(t, x_{i}, y_{i}\right)-u_{\mathrm{ex}}\left(t, x_{i}, y_{i}\right)\right|
\end{aligned}
$$

The best results using $\Delta t=10^{-5}$ and $101 \times 101 \mathrm{RBFs}$ obtained in [20] are shown in Table XVI 13 (Table 12 in [20]). Comparing the results in Tables XV and XVI, we observe that our results are slightly more accurate using only $K=50$. Furthermore, in [20] the authors indicated that their 
Table XV. $\left\|u-u_{\mathrm{ex}}\right\|_{\text {avg }}$ and $\left\|u-u_{\mathrm{ex}}\right\|_{\infty}$ in Example 7 using the Lanczos regularization in a squared domain.

\begin{tabular}{|c|c|c|c|c|c|c|}
\hline \multirow[b]{2}{*}{$t$} & \multicolumn{2}{|c|}{$\Delta t=10^{-5}$} & \multicolumn{2}{|c|}{$\Delta t=10^{-4}$} & \multicolumn{2}{|c|}{$\Delta t=10^{-3}$} \\
\hline & $\left\|u-u_{\mathrm{ex}}\right\|_{\text {avg }}$ & $\left\|u-u_{\mathrm{ex}}\right\|_{\infty}$ & $\left\|u-u_{\mathrm{ex}}\right\|_{\text {avg }}$ & $\left\|u-u_{\mathrm{ex}}\right\|_{\infty}$ & $\left\|u-u_{\mathrm{ex}}\right\|_{\text {avg }}$ & $\left\|u-u_{\mathrm{ex}}\right\|_{\infty}$ \\
\hline 0.001 & $2.9 \times 10^{-4}$ & $3.8 \times 10^{-3}$ & $7.9 \times 10^{-4}$ & $9.7 \times 10^{-3}$ & - & - \\
\hline 0.01 & $4.3 \times 10^{-5}$ & $2.0 \times 10^{-4}$ & $4.3 \times 10^{-4}$ & $1.8 \times 10^{-3}$ & $4.4 \times 10^{-3}$ & $1.8 \times 10^{-2}$ \\
\hline 0.1 & $1.7 \times 10^{-5}$ & $7.9 \times 10^{-5}$ & $1.1 \times 10^{-4}$ & $2.1 \times 10^{-4}$ & $1.1 \times 10^{-3}$ & $1.9 \times 10^{-3}$ \\
\hline 1 & $3.3 \times 10^{-6}$ & $1.1 \times 10^{-6}$ & $2.1 \times 10^{-6}$ & $5.9 \times 10^{-6}$ & $1.2 \times 10^{-5}$ & $3.1 \times 10^{-5}$ \\
\hline 2 & - & - & - & - & $9.5 \times 10^{-8}$ & $2.5 \times 10^{-7}$ \\
\hline 5 & - & - & - & - & $4.3 \times 10^{-14}$ & $1.1 \times 10^{-13}$ \\
\hline
\end{tabular}

Table XVI. $\left\|u-u_{\mathrm{ex}}\right\|_{\text {avg }}$ and $\left\|u-u_{\mathrm{ex}}\right\|_{\infty}$ in Example 7 by Šarler and Vertnik [20].

\begin{tabular}{lll}
\hline & \multicolumn{2}{c}{$\Delta t=10^{-5}$} \\
\cline { 2 - 3 }$t$ & $\left\|u-u_{\mathrm{ex}}\right\|_{\mathrm{avg}}$ & $\left\|u-u_{\mathrm{ex}}\right\|_{\infty}$ \\
\hline 0.001 & $2.352 \times 10^{-4}$ & $2.809 \times 10^{-3}$ \\
0.01 & $9.371 \times 10^{-5}$ & $3.523 \times 10^{-4}$ \\
0.1 & $9.243 \times 10^{-5}$ & $1.582 \times 10^{-4}$ \\
1 & $8.324 \times 10^{-6}$ & $2.066 \times 10^{-5}$ \\
\hline
\end{tabular}

1 method diverges for the larger time step $\Delta t=10^{-3}$ because the explicit approach is unstable and thus requires very small time step in the time marching scheme (see page 1280 in [20]). The

3 method presented in our paper is implicit and stable for $\Delta t=10^{-3}$ as shown in Table XV. On the other hand, the method proposed in [20] is a local method that has the advantage for solving

5 complicated large-scale problems. Both methods have their own merits for solving different types of problems.

7 5.2. $3 D$ cases

The method described in the previous section can be easily extended to $3 \mathrm{D}$ problems using the

9 approximations in the form

$$
\sum_{\mathbf{m}=1}^{\mathbf{M}} U_{m_{1}, m_{2}, m_{3}} \varphi_{m_{1}}\left(x_{1}\right) \varphi_{m_{2}}\left(x_{2}\right) \varphi_{m_{3}}\left(x_{3}\right)
$$

11 for the approximation of the delta function, the MAFS trial functions, and the solution on each time step.

13 Example 8

We consider the following diffusion equation in $3 \mathrm{D}$ :

$$
\begin{aligned}
\frac{1}{\chi} \frac{\partial u}{\partial t} & =\nabla^{2} u+\frac{a^{2}-r^{2}}{a^{2}}, \quad(x, y, z) \in \Omega, \quad t>0 \\
u(x, y, z, 0) & =0, \quad(x, y, z) \in \Omega \\
u(x, y, z, t) & =0, \quad(x, y, z) \in \partial \Omega, \quad t>0
\end{aligned}
$$


Table XVII. The MSR errors in Example 8 using the Lanzcos regularization.

\begin{tabular}{lcccc}
\hline$t$ & $u_{\mathrm{ex}}(0,0,0, t)$ & $M=15, N=300$ & $M=20, N=400$ & $M=25, N=500$ \\
\hline 0.01 & $8.4 \times 10^{-3}$ & $5.7 \times 10^{-5}$ & $1.5 \times 10^{-6}$ & $5.4 \times 10^{-7}$ \\
0.05 & $2.3 \times 10^{-2}$ & $5.0 \times 10^{-5}$ & $2.5 \times 10^{-6}$ & $5.2 \times 10^{-7}$ \\
0.1 & $2.7 \times 10^{-2}$ & $1.7 \times 10^{-5}$ & $2.5 \times 10^{-6}$ & $2.0 \times 10^{-7}$ \\
0.2 & $2.8 \times 10^{-2}$ & $4.7 \times 10^{-6}$ & $2.5 \times 10^{-6}$ & $1.0 \times 10^{-7}$ \\
0.3 & $2.8 \times 10^{-2}$ & $4.3 \times 10^{-6}$ & $2.5 \times 10^{-6}$ & $9.9 \times 10^{-8}$ \\
\hline
\end{tabular}

Table XVIII. The MSR errors in Example 8 using the Abel regularization.

\begin{tabular}{lcccc}
\hline$t$ & $\mathrm{u}_{\mathrm{ex}}(0,0,0, t)$ & $M=15, N=300$ & $M=20, N=400$ & $M=25, N=500$ \\
\hline 0.01 & $8.4 \times 10^{-3}$ & $1.7 \times 10^{-4}$ & $6.7 \times 10^{-5}$ & $9.2 \times 10^{-6}$ \\
0.05 & $2.3 \times 10^{-2}$ & $6.3 \times 10^{-4}$ & $6.0 \times 10^{-5}$ & $1.7 \times 10^{-5}$ \\
0.1 & $2.7 \times 10^{-2}$ & $7.3 \times 10^{-4}$ & $3.0 \times 10^{-5}$ & $1.9 \times 10^{-5}$ \\
0.2 & $2.8 \times 10^{-2}$ & $7.4 \times 10^{-4}$ & $1.9 \times 10^{-5}$ & $2.0 \times 10^{-5}$ \\
0.3 & $2.8 \times 10^{-2}$ & $7.4 \times 10^{-4}$ & $1.9 \times 10^{-5}$ & $2.0 \times 10^{-5}$ \\
\hline
\end{tabular}

1 where $\Omega \cup \partial \Omega=\left\{(x, y, z): x^{2}+y^{2}+z^{2} \leqslant a^{2}\right\}, r^{2}=x^{2}+y^{2}+z^{2}$, and $\chi$ is the diffusion coefficient.

The exact solution is given by [23]

$$
u_{\mathrm{ex}}=\frac{\left(a^{2}-r^{2}\right)\left(7 a^{2}-3 r^{2}\right)}{60 a^{2}}-\frac{12 a^{3}}{r \pi^{5}} \sum_{n=1}^{\infty} \frac{(-1)^{n-1}}{n^{5}} \sin \frac{n \pi r}{a} \exp \left(-\frac{\chi n^{2} \pi^{2} t}{a^{2}}\right)
$$

In the following numerical computation, we consider $a=0.5, \chi=1$. To demonstrate that our 5 proposed approach can be easily extended to 3D problems, we choose to solve this problem in the Cartesian coordinates without considering the special property of its spherical symmetry.

$7 \quad$ For all the numerical results obtained in the example, the number of source points $K$ is equal to the number of collocation points $N$. These points are randomly distributed using the subroutine

9 RNSPH from the IMSL Library. The source points are placed on the sphere with radius $R_{S}=0.95$. In all the calculations performed in this example the number of Chebyshev's polynomials in each 11 axis direction is $N_{x}=N_{y}=N_{z}=5$. A total of 50 test points inside the sphere are randomly selected.

Table XVII contains the MSR solution errors on a set of test points that are distributed uniformly 13 inside the sphere. The results are obtained by the Lanzcos regularization technique. We used the time step $\Delta t=0.01$ for $M=15,20$ and $\Delta t=0.001$ for $M=25$.

15 In Table XVIII, we show some of the numerical results using Abel's regularization technique. We use the time step $\Delta t=0.01$ for $M=15,20$ and $\Delta t=0.001$ for $M=25$. The regularization

17 parameter is $\alpha=0.02$ for $M=15$ and $\alpha=0.01$ for $M=20,25$. In this example, numerical results obtained using Lanzcos regularization scheme seems superior than those from Abel's regularization 19 scheme. Both schemes produce excellent results. 


\section{CONCLUDING REMARKS}

The numerical technique presented in this paper can be classified as a boundary meshless method.

3 The Chebyshev polynomial and trigonometric basis functions for the evaluation of approximate particular solution [5] are coupled with the approximate fundamental solution for finding the

5 corresponding homogeneous solution [4]. We extend these novel approaches to solve the heat conduction problems.

7 In the past, the fundamental solution has been used as the trial function for the approximation of homogeneous solution and the radial basis functions have been widely used as the trial function

9 for the approximation of particular solution. One special feature presented in this paper is that we apply the same trial function for the approximation of fundamental solution and particular solution.

11 Since the particular solution and approximate fundamental solution are easy to derive using the proposed trial function, the same numerical scheme can be extended to a large class of linear

13 PDEs. The proposed method is highly accurate. Hence, we expect other types of linear or nonlinear time-dependent problems such as wave equations, Burger equation, convection-diffusion

15 equations, etc. can be solved effectively using the proposed technique. Further work in extending our approach beyond the heat conduction problems is currently under investigation.

17 One of the challenges of the proposed approach is the optimal choice of the various parameters. The excellent numerical results in this paper merit further investigation in this respect.

\section{REFERENCES}

1. Fairweather G, Karageorghis A. The method of fundamental solutions for elliptic boundary value problems. Advances in Computational Mathematics 1998; 9:69-95.

2. Golberg MA, Chen CS. The method of fundamental solutions for potential, Helmholtz and diffusion problems. In Boundary Integral Methods-Numerical and Mathematical Aspects, Golberg MA (ed.). Computational Mechanics

\section{$23 \quad$ Boundary Integral Methods-Nu}

25 3. Cho HA, Golberg MA, Muleshkov AS, Li X. Trefftz methods for time dependent partial differential equations. CMC 2004; 1:1-37.

27 4. Reutskiy S. A boundary method of Trefftz type with approximate trial functions. Engineering Analysis with Boundary Elements 2002; 26(4):341-353.

29 5. Reutskiy S, Chen CS. Approximation of multivariate functions and evaluation of particular solutions using Chebyshev polynomial and trigonometric basis functions. International Journal for Numerical Methods in Engineering 2006; 67:1811-1829.

6. Young DL, Tsai CC, Fan CM. Direct approach to solve nonhomogeneous diffusion problems using fundamental solutions and dual reciprocity methods. Journal of the Chinese Institute of Engineers 2004; 27:597-609.

7. Zhu S, Satravaha P, Lu X. Solving linear diffusion equations with the dual reciprocity method in Laplace space. Engineering Analysis with Boundary Elements 1995; 13(1):1-10.

8. Chen CS, Rashed YF, Golberg MA. A mesh free method for linear diffusion equations. Numerical Heat Transfer, Part B 1998; 33:469-486.

9. Li X, Ho CH, Chen CS. Computational test of approximation of functions and their derivatives by radial basis functions. Neural, Parallel and Scientific Computations 2002; 10:25-46.

10. Ingber M, Chen CS, Tanski JA. A mesh free approach using radial basis functions and parallel domain decomposition for solving three dimensional diffusion equations. International Journal of Numerical Methods in

\section{$41 \quad \frac{\text { decomposition for solving three di }}{\text { Engineering 2004; 60:2183-2201. }}$}

43 11. Chen W. Symmetric boundary knot method. Engineering Analysis with Boundary Elements 2002; 26:489-494.

12. Lanczos C. Applied Analysis. Prentice-Hall: Englewood Cliffs, NJ, 1956.

45 13. Reutskiy S. A Trefftz type method for time-dependent problems. Engineering Analysis with Boundary Elements 2004; 28:13-21.

47 14. Reutskiy S. A boundary method of Trefftz type for PDEs with scattered data. Engineering Analysis with Boundary Elements 2005; 29:713-724. 
1 15. Available from: http://www.answers.com/topic/zeta-function-regularization.

16. Ramachandran PA, Balakrishnan K. Radial basis functions as approximate particular solutions: review of recent progress. Engineering Analysis with Boundary Elements 2000; 24:575-582.

17. Boyd JP. Chebyshev and Fourier Spectral Methods. Dover: New York, 2001.

5 18. Bernardi C, Maday Y. Spectral methods. In Handbook of Numerical Analysis, vol. V, Ciarlet PG, Lions J-L (eds). 1997; 209-485.

7 19. Canuto C, Hussaini MY, Quarteroni A, Zang TA. Spectral Methods in Fluid Dynamics. Springer: New York, 1988.

9 20. Šarler B, Vertnik R. Meshfree explicit local radial basis function collocation method for diffusion problems. Computers and Mathematics with Applications 2006; 51:1269-1282.

11 21. Franke R. Scattered data interpolation: test of some methods. Mathematics of Computation 1982; 48:181-200. 22. MathWorks: Developers of MATLAB and Simulink. Available from: http://www.mathworks.com.

13 23. Carslaw HS, Jaeger JC. Conduction of Heat in Solids. Oxford University Press: London, 1995. 
John Wiley \& Sons Ltd

WILEY

\section{Author Queries For NME 2230}

While preparing this paper/manuscript for typesetting, the following queries have arisen

\begin{tabular}{|c|c|l|l|}
\hline $\begin{array}{c}\text { Query } \\
\text { No. }\end{array}$ & $\begin{array}{c}\text { Proof } \\
\text { Page/line } \\
\text { no }\end{array}$ & Details required & Authors response \\
\hline 1 & References & Please provide the place of publisher for Ref. [2]. & \\
\hline 2 & References & Please provide the name of publisher and place for Ref. [18]. & \\
\hline & & & \\
\hline & & & \\
\hline & & & \\
\hline & & & \\
\hline & & & \\
\hline & & & \\
\hline & & & \\
\hline & & & \\
\hline
\end{tabular}


(the "Contribution") written by

(the "Contributor") for publication in..

(the "Journal) published by John Wiley \& Sons Ltd ("Wiley").

In order to expedite the publishing process and enable Wiley to disseminate your work to the fullest extent, we need to have this Copyright Transfer Agreement signed and returned to us with the submission of your manuscript. If the Contribution is not accepted for publication this Agreement shall be null and void.

\section{A. COPYRIGHT}

1. The Contributor assigns to Wiley, during the full term of copyright and any extensions or renewals of that term, all copyright in and to the Contribution, including but not limited to the right to publish, republish, transmit, sell, distribute and otherwise use the Contribution and the material contained therein in electronic and print editions of the Journal and in derivative works throughout the world, in all languages and in all media of expression now known or later developed, and to license or permit others to do so.

2. Reproduction, posting, transmission or other distribution or use of the Contribution or any material contained therein, in any medium as permitted hereunder, requires a citation to the Journal and an appropriate credit to Wiley as Publisher, suitable in form and content as follows: (Title of Article, Author, Journal Title and Volume/Issue Copyright $\odot$ [year] John Wiley \& Sons Ltd or copyright owner as specified in the Journal.)

\section{B. RETAINED RIGHTS}

Notwithstanding the above, the Contributor or, if applicable, the Contributor's Employer, retains all proprietary rights other than copyright, such as patent rights, in any process, procedure or article of manufacture described in the Contribution, and the right to make oral presentations of material from the Contribution.

\section{OTHER Rights OF CONTRIBUtOR}

Wiley grants back to the Contributor the following:

1. The right to share with colleagues print or electronic "preprints" of the unpublished Contribution, in form and content as accepted by Wiley for publication in the Journal. Such preprints may be posted as electronic files on the Contributor's own website for personal or professional use, or on the Contributor's internal university or corporate networks/intranet, or secure external website at the Contributor's institution, but not for commercial sale or for any systematic external distribution by a third party (eg: a listserver or database connected to a public access server). Prior to publication, the Contributor must include the following notice on the preprint: "This is a preprint of an article accepted for publication in [Journal title] Copyright $\odot$ (year) (copyright owner as specified in the Journal)". After publication of the Contribution by Wiley, the preprint notice should be amended to read as follows: "This is a preprint of an article published in [include the complete citation information for the final version of the Contribution as published in the print edition of the Journal]" and should provide an electronic link to the Journal's WWW site, located at the following Wiley URL: http://www.interscience.wiley.com/. The Contributor agrees not to update the preprint or replace it with the published version of the Contribution.

2. The right, without charge, to photocopy or to transmit on-line or to download, print out and distribute to a colleague a copy of the published Contribution in whole or in part, for the Contributor's personal or professional use, for the advancement of scholarly or scientific research or study, or for corporate informational purposes in accordance with paragraph D2 below.

3. The right to republish, without charge, in print format, all or part of the material from the published Contribution in a book written or edited by the Contributor

4. The right to use selected figures and tables, and selected text (up to 250 words) from the Contribution, for the Contributor's own teaching purposes, or for incorporation within another work by the Contributor that is made part of an edited work published (in print or electronic format) by a third party, or for presentation in electronic format on an internal computer network or external website of the Contributor or the Contributor's employer. The abstract shall not be included as part of such selected text.

5. The right to include the Contribution in a compilation for classroom use (course packs) to be distributed to students at the Contributor's institution free of charge or to be stored in electronic format in datarooms for access by students at the Contributor's institution as part of their course work (sometimes called "electronic reserve rooms") and for in-house training programmes at the Contributor's employer.

\section{CONTRIBUTIONS OWNED BY EMPLOYER}

1. If the Contribution was written by the Contributor in the course of the Contributor's employment (as a "work-made-for-hire" in the course of employment), the Contribution is owned by the company/employer which must sign this Agreement (in addition to the Contributor's signature), in the space provided below. In such case, the company/employer hereby assigns to Wiley, during the full term of copyright, all copyright in and to the Contribution for the full term of copyright throughout the world as specified in paragraph A above.

2. In addition to the rights specified as retained in paragraph B above and the rights granted back to the Contributor pursuant to paragraph $\mathrm{C}$ above, Wiley hereby grants back, without charge, to such company/employer, its subsidiaries and divisions, the right to make copies of and distribute the published Contribution internally in print format or electronically on the Company's internal network. Upon payment of the Publisher's reprint fee, the institution may distribute (but not re-sell) print copies of the published Contribution externally. Although copies so made shall not be available for individual re-sale, they may be included by the company/employer as part of an information package included with software or other products offered for sale or license. Posting of the published Contribution by the institution on a public access website may only be done with Wiley's written permission, and payment of any applicable fee(s). 


\section{E. GOVERNMENT CONTRACTS}

In the case of a Contribution prepared under US Government contract or grant, the US Government may reproduce, without charge, all or portions of the Contribution and may authorise others to do so, for official US Government purposes only, if the US Government contract or grant so requires. (Government Employees: see note at end.)

\section{F. COPYRIGHT Notice}

The Contributor and the company/employer agree that any and all copies of the Contribution or any part thereof distributed or posted by them in print or electronic format as permitted herein will include the notice of copyright as stipulated in the Journal and a full citation to the Journal as published by Wiley.

\section{G. CONTRIBUTOR'S REPRESENTATIONS}

The Contributor represents that the Contribution is the Contributor's original work. If the Contribution was prepared jointly, the Contributor agrees to inform the coContributors of the terms of this Agreement and to obtain their signature(s) to this Agreement or their written permission to sign on their behalf. The Contribution is submitted only to this Journal and has not been published before, except for "preprints" as permitted above. (If excerpts from copyrighted works owned by third parties are included, the Contributor will obtain written permission from the copyright owners for all uses as set forth in Wiley's permissions form or in the Journal's Instructions for Contributors, and show credit to the sources in the Contribution.) The Contributor also warrants that the Contribution contains no libelous or unlawful statements, does not infringe on the right or privacy of others, or contain material or instructions that might cause harm or injury.

Tick one box and fill in the appropriate section before returning the original signed copy to the Publisher

\section{Contributor-owned work}

Contributor's signature Date

Type or print name and title

Co-contributor's signature Date

Type or print name and title

\section{Attach additional signature page as necessary}

Company/Institution-owned work (made-for-

hire in the course of employment)

Contributor's signature

Type or print name and title

Company or Institution

(Employer-for Hire)

Authorised signature of Employer

Date

Type or print name and title

\section{US Government work}

Note to US Government Employees

A Contribution prepared by a US federal government employee as part of the employee's official duties, or which is an official US Government publication is called a "US Government work", and is in the public domain in the United States. In such case, the employee may cross out paragraph A1 but must sign and return this Agreement. If the Contribution was not prepared as part of the employee's duties or is not an official US Government publication, it is not a US Government work.

\section{UK Government work (Crown Copyright)}

Note to UK Government Employees

The rights in a Contribution by an employee of a UK Government department, agency or other Crown body as part of his/her official duties, or which is an official government publication, belong to the Crown. In such case, the Publisher will forward the relevant form to the Employee for signature. 


\section{WILEY AUTHOR DISCOUNT CARD}

As a highly valued contributor to Wiley's publications, we would like to show our appreciation to you by offering a unique $\mathbf{2 5 \%}$ discount off the published price of any of our books*.

To take advantage of this offer, all you need to do is apply for the Wiley Author Discount Card by completing the attached form and returning it to us at the following address:

The Database Group

John Wiley \& Sons Ltd

The Atrium

Southern Gate

Chichester

West Sussex P019 8SQ

UK

In the meantime, whenever you order books direct from us, simply quote promotional code S001W to take advantage of the $25 \%$ discount.

The newest and quickest way to order your books from us is via our new European website at:

\section{http://www.wileyeurope.com}

Key benefits to using the site and ordering online include:

- $\quad$ Real-time SECURE on-line ordering

- The most up-to-date search functionality to make browsing the catalogue easier

- Dedicated Author resource centre

- E-mail a friend

- $\quad$ Easy to use navigation

- Regular special offers

- $\quad$ Sign up for subject orientated e-mail alerts

So take advantage of this great offer, return your completed form today to receive your discount card.

Yours sincerely,

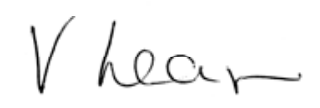

Verity Leaver

E-marketing and Database Manager 


\section{REGISTRATION FORM FOR 25\% BOOK DISCOUNT CARD}

To enjoy your special discount, tell us your areas of interest and you will receive relevant catalogues or leaflets from which to select your books. Please indicate your specific subject areas below.

Accounting

- Public

- Corporate

\section{Chemistry}

- Analytical

- Industrial/Safety

- Organic

- Inorganic

- Polymer

- Spectroscopy

Encyclopedia/Reference

- Business/Finance

- Life Sciences

- Medical Sciences

- Physical Sciences

- Technology

\section{Earth \& Environmental Science}

Hospitality

\section{Genetics}

- Bioinformatics/Computational Biology

- Proteomics

- Genomics

- Gene Mapping

- Clinical Genetics

\section{Medical Science}

- Cardiovascular

- Diabetes

- Endocrinology

- Imaging

- Obstetrics/Gynaecology

- Oncology

- Pharmacology

- Psychiatry

Non-Profit

\section{Architecture}

Business/Management

Computer Science

- Database/Data Warehouse

- Internet Business

- Networking

- Programming/Software Development

- Object Technology

Engineering

- Civil

- Communications Technology

- Electronic

- Environmental

- Industrial

- Mechanical

Finance/Investing

- Economics

- Institutional

- Personal Finance

Life Science

Landscape Architecture

Mathematics/Statistics

Manufacturing

Material Science

Psychology

- Clinical

- Forensic

- Social \& Personality

- Health \& Sport

- Cognitive

- Organizational

- Developmental and Special Ed

- Child Welfare

- Self-Help

Physics/Physical Science 
SIGNATURE:

PLEASE COMPLETE THE FOLLOWING DETAILS IN BLOCK CAPITALS:

TITLE AND NAME: (e.g. Mr, Mrs, Dr)

JOB TITLE:

DEPARTMENT:

COMPANY/INSTITUTION:

ADDRESS:

TOWN/CITY:

COUNTY/STATE:

COUNTRY:

POSTCODE/ZIP CODE:

DAYTIME TEL:

FAX:

E-MAIL:

YOUR PERSONAL DATA

We, John Wiley \& Sons Ltd, will use the information you have provided to fulfil your request. In addition, we would like to:

1. Use your information to keep you informed by post, e-mail or telephone of titles and offers of interest to you and available from us or other Wiley Group companies worldwide, and may supply your details to members of the Wiley Group for this purpose.

[ ] Please tick the box if you do not wish to receive this information

2. Share your information with other carefully selected companies so that they may contact you by post, fax or e-mail with details of titles and offers that may be of interest to you.

[ ] Please tick the box if you do not wish to receive this information.

If, at any time, you wish to stop receiving information, please contact the Database Group (databasegroup@wiley.co.uk) at John Wiley \& Sons Ltd, The Atrium, Southern Gate, Chichester, West Sussex PO19 8SQ, UK.

\section{E-MAIL ALERTING SERVICE}

We offer an information service on our product ranges via e-mail. If you do not wish to receive information and offers from John Wiley companies worldwide via e-mail, please tick the box [ ].

This offer is exclusive to Wiley Authors, Editors, Contributors and Editorial Board Members in acquiring books (excluding encyclopaedias and major reference works) for their personal use. There should be no resale through any channel. The offer is subject to stock availability and may not be applied retrospectively. This entitlement cannot be used in conjunction with any other special offer. Wiley reserves the right to vary the terms of the offer at any time.

Ref: S001W 